\title{
In Vitro Adsorption of Analgosedative Drugs in New Extracorporeal Membrane Oxygenation Circuits
}

\author{
Genny Raffaeli, $\mathrm{MD}^{1,2}$; Karel Allegaert, $\mathrm{MD}, \mathrm{PhD}^{1}$; Birgit Koch, $\mathrm{MD}^{3}$; Giacomo Cavallaro, $\mathrm{MD}, \mathrm{PhD}^{2}$; \\ Fabio Mosca, $\mathrm{MD}^{2}$; Dick Tibboel, MD, $\mathrm{PhD}^{1}$; Enno D. Wildschut, MD, $\mathrm{PhD}^{1}$
}

\begin{abstract}
Objective: Evaluate drug disposition of sedatives and analgesics in the Xenios/Novalung extracorporeal membrane oxygenation circuits.

Design: In vitro experimental study.

Setting: Erasmus MC - Sophia Children's Hospital, Rotterdam, The Netherlands.
\end{abstract}

Subjects: Nine closed-loop extracorporeal membrane oxygenation circuits, made up of the iLA Activve console with four different iLA Activve kits: two X-lung kits, two iLA-Activve iLA kits, two MiniLung kits, and three MiniLung petite kits.

Interventions: The circuits were primed with fresh whole blood and maintained under physiologic conditions ( $\mathrm{pH} /$ temperature) throughout 24 hours. Paracetamol, morphine, midazolam, fentanyl, and sufentanil were injected as standard age-related doses into nine closed-loop extracorporeal membrane oxygenation circuits.

Measurements and Main Results: Pre-membrane (P2) blood samples were obtained prior to drug injection and after injection at $2,10,30,180,360$ minutes, and at 24 hours. A control sample at 2 minutes was collected for spontaneous drug degradation testing at 24 hours. Two hundred sixteen samples were analyzed. After correction for the spontaneous drug degradation, the mean drug loss at 24 hours was paracetamol 49\%, morphine 51\%, midazolam 40\%, fentanyl 84\%, sufentanil 83\%. Spontaneous deg-

\footnotetext{
'Intensive Care and Department of Pediatric Surgery, Erasmus MC Sophia Children's Hospital, Rotterdam, The Netherlands.

${ }^{2}$ Neonatal Intensive Care Unit, Department of Clinical Sciences and Community Health, Fondazione IRCCS Cà Granda Ospedale Maggiore Policlinico, Università degli Studi di Milano, Milan, Italy.

${ }^{3}$ Department of Hospital Pharmacy, Erasmus MC, Rotterdam, The
} Netherlands.

This study was performed at Erasmus MC - Sophia Children's Hospital, Intensive Care and Department of Pediatric Surgery, Rotterdam, The Netherlands.

Supported by unrestricted research grant from Xenios Germany. Dr. Raffaeli received a research grant from "A.Griffini - J. Miglierina 2013 Foundation."

Dr. Wildschut's institution received funding from Xenios. The remaining authors have disclosed that they do not have any potential conflicts of interest.

For information regarding this article, E-mail: genny.raffaeli@gmail.com

Copyright (C) 2018 by the Society of Critical Care Medicine and the World Federation of Pediatric Intensive and Critical Care Societies

DOI: $10.1097 /$ PCC.0000000000001484 radation was paracetamol $6 \%$, morphine $0 \%$, midazolam $11 \%$, fentanyl $4 \%$, and sufentanil $0 \%$. The decline of drug concentration over time was more pronounced for the more lipophilic drugs.

Conclusions: Loss of highly lipophilic drugs in the extracorporeal membrane oxygenation circuits at 24 hours was remarkable. Drug loss is comparable with other hollow fiber extracorporeal membrane oxygenation systems but less than in silicone-based membranes especially in the first hours after injection. (Pediatr Crit Care Med 2018; XX:00-00)

Key Words: analgesics; drug disposition; extracorporeal membrane oxygenation; pharmacokinetics; sedatives

xtracorporeal membrane oxygenation (ECMO) is a type $\checkmark$ of cardiopulmonary bypass (CPB) designed to temporarily support respiratory and/or cardiac function in critically ill patients, who have failed conventional treatment (1).

In these patients, adequate analgosedation is necessary to provide comfort and pain relief but also to prevent complications related to excessive movement, such as cannula dislocation, impaired ECMO flow or unintentional decannulation (2). Traditionally, deep sedation was pursued, while current trends, given the sedation-associated morbidity, are moving toward minimal sedation and "awake ECMO" strategies $(3,4)$. Interruption of sedatives and analgesics was proved to be feasible and safe in ECMO patients, across all age groups (5-7). To reach the desired target level of analgosedation, different classes of drugs are available, with opioids and benzodiazepines as the first choice in most centers (2). Based on clinical monitoring and pain assessment by validated scales, these medications are usually titrated to the desired effect (2). Prolonged administration may be associated with serious adverse effects (i.e., tolerance, physiologic dependence), contributing to potential development of withdrawal syndrome, in case of abrupt discontinuation (8). Iatrogenic abstinence by itself will impact negatively on patient outcome, by increasing hospital stay, resource allocation, and overall health costs (9-11). Therefore, rational drug dosing is needed and, subsequently, a better understanding of pharmacokinetic (PK) variability on ECMO is required. 
It is known that developmental changes related to body composition, protein binding (PB), organ (especially liver and kidney) function largely affect drugs' PK (12). Nonmaturational covariates (such as critical illness, inflammation, pharmacogenetics) add specific variability to the complexity of pediatric pharmacotherapy (13).

Besides developmental aspects, drug-specific and pathology-related PK changes, the ECMO equipment further complicates the issue $(12,14)$. Several studies show that PK is altered during ECMO with increased volume of distribution $(\mathrm{Vd})$ and changes in clearance compared with non-ECMO patients. Major determinants of increased Vd are adsorption of drugs within the ECMO circuit, supplements of fluids, and blood products $(14,15)$. The extent of sequestration to various components depends on the physicochemical characteristics of a drug, such as molecular size, drug stability, plasma PB, and lipophilicity (16-19). Additional variability is associated with ECMO system-related factors, including the type of pump and oxygenator, circuit size, and coating of the tubing (20-22).

Despite the wide use of sedatives and analgesics during ECMO, evidence-based dosing guidelines are largely lacking. Although morphine, fentanyl, and midazolam have been investigated in previous mainly neonatal PK studies (23-25), recent changes in ECMO technology require new insights. Sparse data on drug disposition in newer systems made up of microporous membranes are available $(19,26,27)$.

Newer ECMO systems, such as Xenios-Novalung (Heilbronn, Germany), may reveal significant differences in drug disposition compared with older systems, which might influence efficacy and safety of pharmacotherapy in clinical practice. As pain and sedation management is imperative to provide effective ECMO support in all age groups, special efforts should be addressed in order to provide data on drugspecific ECMO-related PK parameters, promoting evidencebased prescription to ensure quality of care and safety.

To this aim, we set up nine different in vitro closed-loop circuit models to evaluate potential effects of Xenios-Novalung systems on morphine, fentanyl, sufentanil, paracetamol, and midazolam.

\section{MATERIALS AND METHODS}

The study was performed at the ICU of the Sophia Children's Hospital, Erasmus MC in Rotterdam, The Netherlands. The need for the institutional review board approval was waived, according to the Dutch Law of research on human subjects this type of investigation in which no patients are involved are not subject to ethical approval.

The studied circuits were made available to us by the Xenios company as part of an unrestricted research grant.

\section{ECMO Circuits}

Nine different in vitro models of ECMO circuits were set up, consisting of four different $i L A$ Activve kits: two iLA-Activve $X$-lung kits, two iLA-Activve iLA kits, two iLA-Activve MiniLung kits and three iLA-Activve MiniLung petite kits. All are hollow fiber oxygenators with a diffusion membrane for adult, pediatric and neonatal patients. Two test runs per group were carried out, except for neonatal circuit, in which we performed three tests. Each circuit was first primed according to local clinical protocol with Ringer's lactate solution, albumin $20 \%(15 \mathrm{~mL})$ and sodium bicarbonate $8.4 \%(4 \mathrm{~mL})$; then it was allowed to circulate for 10 minutes before being "blood primed" with whole blood. Heparin bolus of 500 UI was added to the system to prevent clotting. Activated clotting time (ACT) values were maintained for more than 200 seconds. Temperature, hematocrit, and $\mathrm{pH}$ values were maintained within normal ranges during the study period; sweep gas or sodium bicarbonate solution was added to the circuit to maintain the $\mathrm{pH}$ in the range of 7.25-7.50. Blood gas/electrolyte analysis and ACT were performed using an Abbott $i$-STAT portable analyser (Abbott Labs, Lake Bluff, IL). Temperature was maintained at $37.5^{\circ} \mathrm{C}$ using the incorporated heater in the iLA-Activve MiniLung, iLA-Activve MiniLung petite and iLAActivve $X$-lung membranes, whereas external heating was used in the iLA-Activve iLA membrane with target temperatures between $36^{\circ} \mathrm{C}$ and $37.9^{\circ} \mathrm{C}$. Circuits were made continuous by connecting the ends of the arterial and venous cannulas to a small reservoir bag containing $100 \mathrm{~mL}$ of priming fluid, allowing a continuous flow of priming fluid throughout the entire circuit. The volume of the neonatal, infant, pediatric, and adult ECMO circuits are estimated at 225, 280, 360, and $400 \mathrm{~mL}$, respectively. The ECMO circuit was filled to maximal capacity. Before drug injection, an equal volume of fluid was subtracted from the ECMO circuit. Flow rates were set to run for 24 hours at a flow rate of $500 \mathrm{~mL} / \mathrm{min}$ (neonatal circuit), $700 \mathrm{~mL} / \mathrm{min}$ (infant), $2.5 \mathrm{~L} / \mathrm{min}$ (pediatric), $3.5 \mathrm{~L} / \mathrm{min}$ (adult).

\section{Drug Administration}

Drugs were injected at 5-second intervals through a line that was connected to the tubing right after the reservoir bag (P1) simulating actual drug administration in clinical setting. The line was flushed with $1 \mathrm{~mL}$ of physiologic saline solution $(0.9 \%)$ inbetween injections of the different drugs in order to avoid crystallization or pooling effects. Drugs were dosed according to a standardized weight for a newborn $(3.5 \mathrm{~kg})$. Drug dosing for the other systems were increased in proportion to the increase in volume to achieve similar theoretical concentrations. The order of drug injection was the following (neonatal/infant/ pediatric/adult): paracetamol $60 \mathrm{mg} / 70 \mathrm{mg} / 90 \mathrm{mg} / 119 \mathrm{mg}$, morphine $0.5 \mathrm{mg} / 0.6 \mathrm{mg} / 0.75 \mathrm{mg} / 1.02 \mathrm{mg}$, sufentanil $5 \mu \mathrm{g} /$ $6 \mu \mathrm{g} / 7.5 \mu \mathrm{g} / 10.2 \mu \mathrm{g}$, midazolam $0.5 \mathrm{mg} / 0.6 \mathrm{mg} / 0.75 \mathrm{mg} / 1.02 \mathrm{mg}$, fentanyl $15 \mu \mathrm{g} / 18 \mu \mathrm{g} / 22.5 \mu \mathrm{g} / 30.6 \mu \mathrm{g}$.

\section{Samples}

Once the circuit was primed, before drugs injection, a premembrane (P2) sample (T0) was obtained from a line attached to the tubing prior to the reservoir bag, in order to document the absence of any of the drugs tested in the priming fluid. During the circuit run, premembrane (P2) samples were drawn at various time intervals throughout the 24-hour in vitro testing (i.e., 2, 10, 30, 180, 360 minutes, and 24 hours after injection). Two minutes after the injection, a control sample was collected 
(T2-24), stored externally in a ethylenediaminetetraacetate (EDTA) sample tube, exposed to outside light and maintained at the same temperature as the tested circuits. The samples were analyzed at 24 hours to determine spontaneous drug degradation. Whole blood was collected in polypropylene tubes containing EDTA and stored at $4^{\circ} \mathrm{C}$ for a maximum of 12 hours until further processing. Thereafter blood samples were centrifuged (6 min at 3,000 round per min) after which the plasma supernatant was transferred to clean labeled polypropylene cryogenic vials with polyethylene screw caps (Nalgene Labware, Rochester, $\mathrm{NY}$ ). Plasma samples were stored at $-80^{\circ} \mathrm{C}$ until drug analysis.

Paracetamol, morphine, midazolam, fentanyl, and sufentanil were analyzed by means of ultrafast liquid chromatographymass spectrometry (LC-MS/MS) in the pharmacy laboratory of Erasmus MC (28). Methods were validated according to U.S. Food and Drug Administration guidelines for bioanalytical method validation (29). Thermo Vantage LC-MS/MS equipment was used for paracetamol and midazolam. For fentanyl and morphine, the UPLC-MS/MS system was purchased from Waters Chromatography B.V. (Etten-Leur, The Netherlands) and was composed of Waters Acquity UPLC Sample Manager (Ser. No. H08UPA597M) and Waters TQ Detector (Ser. No. QBA586). The MassLynx V4.1 SCN627 software package (Waters Corporation, Milford, MA) was used for the acquisition and processing of data. Quantification was performed using QuanLynx as implemented in the software.

Reference values are reported (including lower limit of quantification [LLQ] and upper limit of quantification). Intraand interassay means were within 15\% of the target range value, as requested by the U.S. Food and Drug Administration (FDA).

For morphine (linear calibration range, $1.00-100 \mathrm{ng} / \mathrm{mL}$ ), the within and between-run precisions at five tested concentrations, including the LLQ, were less than or equal to 10.3 and $\leq 8.67 \%$, respectively, whereas the average accuracy ranged from $91.9 \%$ to $96.9 \%$.

For fentanyl and sufentanil (reference range, 0.25-50.00 $\mu \mathrm{g} / \mathrm{L})$, the within and between-run precisions at five tested concentrations, including the LLQ, were less than or equal to 5.52 and less than or equal to $6.12 \%$, respectively, whereas the average accuracy ranged from $88.5 \%$ to $94.0 \%$.
For midazolam (reference range, 2.00-2,400 $\mu \mathrm{g} / \mathrm{L}$ ), intraand interassay means were within $15 \%$ of the target range value, as requested by the FDA.

For paracetamol (reference range, $0.05-25.00 \mathrm{mg} / \mathrm{L}$ ), intraand interassay means were within $15 \%$ of the target range value, as requested by the FDA.

\section{Plasma Drug Concentration and Spontaneous Drug Degradation}

Expected plasma drug concentration was calculated taking into account total circulating volume corrected for adsorption to erythrocytes, using the previously published methods (22), with exception made for sufentanil. Based on LogP values of sufentanil, the plasma ratio is estimated at 75\% comparable with other lipophilic drugs such as midazolam and fentanyl. Drug physicochemical data were derived from the DrugBank online database (30).

Spontaneous drug degradation, that is the amount of drug left after 24 hours of spontaneous decay, was calculated for each compound as a difference in drug recovery between T2 and T2-24. Mean recovery, referred to each circuit category, was corrected by average spontaneous degradation.

Comparison between drugs and four different ECMO circuits (MiniLung petite: MLP, MiniLung: ML, iLA Activve: iLA, Xlung) are presented.

The average recovery and the average recovery corrected by spontaneous drug degradation at 2, 10, 30 minutes, and 3, 6, and 24 hours across all circuit categories were calculated. Trend over time is analyzed and represented graphically for the entire study period for each drug. As the entity of drug loss for the more lipophilic drugs (midazolam, fentanyl, and sufentanil) is predominant in the first 6 hours, the average hourly decay rate is graphically provided for this timeframe. Data are expressed as mean ( \pm SEM) or percentage. MS Excel 2016 was used for all the analyses.

\section{RESULTS}

The in vitro circuits were maintained under physiologic conditions for 24 hours. Throughout the test period, neither circuit complications (i.e., circuit leaks, loss of pump function, interruption to circuit flow), nor loss of drug samples occurred. A total number of 216 samples (24 for each circuit) were

\section{TABLE 1. Mean Drug Recovery ( \pm SEM; in \%) After 24 Hours of Circulation in the Study Testing Circuits, Corrected for Spontaneous Drug Degradation}

\begin{tabular}{|c|c|c|c|c|c|c|c|c|}
\hline Drug & m-All & $\begin{array}{l}\text { m-Minilung } \\
\text { petite }\end{array}$ & m-MiniLung & m-iLA Activve & m-XIung & $\begin{array}{l}\text { m-Spontaneous } \\
\text { Drug } \\
\text { Degradation }\end{array}$ & $\log P$ & $\begin{array}{c}\text { Protein } \\
\text { Binding } \\
(\%)\end{array}$ \\
\hline Midazolam & 60 & $57( \pm 24)$ & $65( \pm 8)$ & $56( \pm 4)$ & $62( \pm 21)$ & 11.4 & 3.89 & 97 \\
\hline Fentanyl & 16 & $18( \pm 3)$ & $10( \pm 3)$ & $20( \pm 2)$ & $16( \pm 6)$ & 0 & 4.12 & $80-85$ \\
\hline Morphine & 49 & $57( \pm 4)$ & $45( \pm 3)$ & $51( \pm 0)$ & $39( \pm 20)$ & 0 & 0.99 & $30-40$ \\
\hline Paracetamol & 51 & $47( \pm 6)$ & $57( \pm 6)$ & $57( \pm 7)$ & $41( \pm 4)$ & 6.4 & 0.51 & 25 \\
\hline Sufentanil & 17 & $6( \pm 2)$ & $8( \pm 1)$ & $16( \pm 4)$ & $38( \pm 20)$ & 0 & 3.4 & $\begin{array}{c}\text { Not } \\
\text { available }\end{array}$ \\
\hline
\end{tabular}

Mean $(m) \%$ of drug recovery ( \pm SEM). Drug loss is corrected for mean spontaneous drug degradation. Mean drug recovery (\%) is shown for each category of circuit. LogP values and protein binding are shown for the study drugs and are derived from Drug Bank (31). 
analyzed. Testing confirmed that all baseline plasma samples were free of study drugs.

Results represent the amount of drug recovery after 24 hours of exposure to the extracorporeal circuit. These values are corrected for average spontaneous drug degradation, and they are reported as mean ( \pm SEM). Negative mean values are expressed as "zero" degradation (Table 1).

The mean percentage of drug loss at 24 hours, corrected for average spontaneous degradation, was $49 \%$ for paracetamol, $40 \%$ for midazolam, $51 \%$ for morphine, $83 \%$ for sufentanil, $84 \%$ for fentanyl. Figure 1 shows average drug recovery plotted against time for each drug, with respect to the four different categories of circuits tested. The decline of drug concentration over time was more pronounced for the more lipophilic drugs (Table 1; Fig. 2). Fentanyl and sufentanil are sequestered for a large fraction of the administered dose in the system, soon after injection, with an average hourly loss rate in the first 6 hours of 13\% (Fig. 2). Differences between ECMO systems (neonatal, infant, pediatric, adult) are most pronounced for sufentanil, whereas other lipophilic drugs' behavior seems to be uniform throughout different sizes.

Spontaneous degradation of drugs was quantified for all study compounds. The mean spontaneous degradation was $11 \%$ for midazolam, $6 \%$ for paracetamol, $4 \%$ for fentanyl, $0 \%$ for both morphine and sufentanil.

\section{DISCUSSION}

This in vitro study provides new insights into equipmentinduced variability in drug disposition of the main analgosedative agents during ECMO support with the iLA-Activve iLA, iLA-Activve MiniLung petite, iLA-Activve MiniLung, and iLAActivve $X$-lung circuits. Lipophilic drugs show ongoing drug adsorption over 24 hours with drug recovery at 24 hours of $16 \%, 17 \%$, and $60 \%$ for fentanyl, sufentanil, and midazolam, respectively. For more hydrophilic drugs, adsorption is less with early steady state concentrations. There does not seem to be any relevant difference between drug adsorption between the different membranes. Spontaneous degradation of drugs over time was limited for the studied drugs.

Analgesia and sedation are challenging issues in the management of critically ill patients on ECMO support. Studies concerning sedation practices during ECMO are few and are focused on mostly used opioids and benzodiazepines $(25,31)$. Little is known about PK of "additional" opioids, like sufentanil or alternative sedatives (32). Several in vitro studies have shown significant drug loss of drugs in circuits $(18,22,33)$. There are substantial differences in drug recovery between ECMO circuits. Especially lipophilic drugs are sequestered by the ECMO circuits resulting in almost $100 \%$ adsorption of midazolam and fentanyl in silicone-based membranes, whereas drug loss is less pronounced in smaller circuits with centrifugal pumps and hollow fiber microporous membranes compared with silicone-based membranes (22). There is a clear relationship between lipophilicity expressed as $\log \mathrm{P}$ and drug adsorption (22). Recently, Shekar et al (19) tested adult ECMO circuits

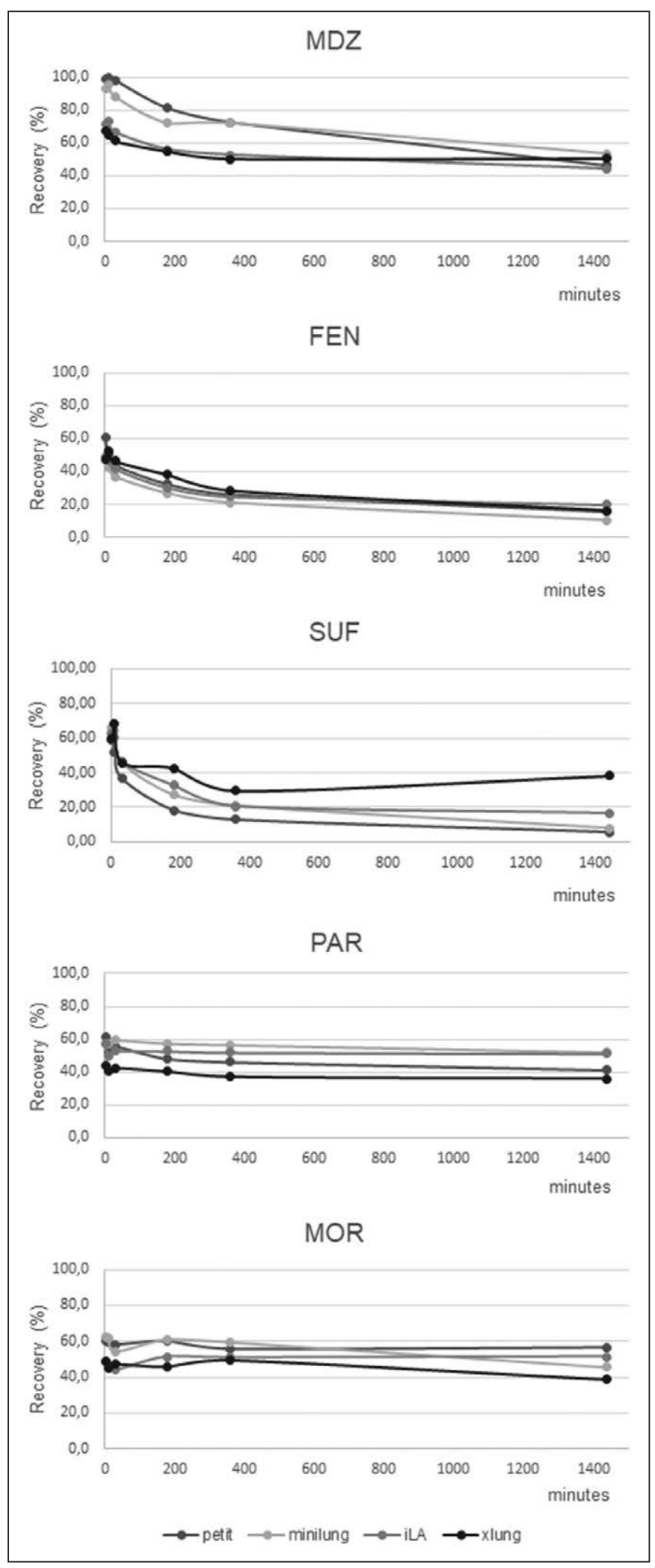

Figure 1. Mean drug recovery (\%) plotted versus time (min) over 24 hours for sedatives and analgesics with respect to the four different categories of circuit tested: Minilung petite (dark gray), MiniLung (light gray), iLa Activve (gray), XLung(black). FEN = fentanyl, $\mathrm{MOR}=$ morphine, $\mathrm{MDZ}=$ midazolam, $\mathrm{PAR}=$ paracetamol, SUF $=$ sufentanil. 


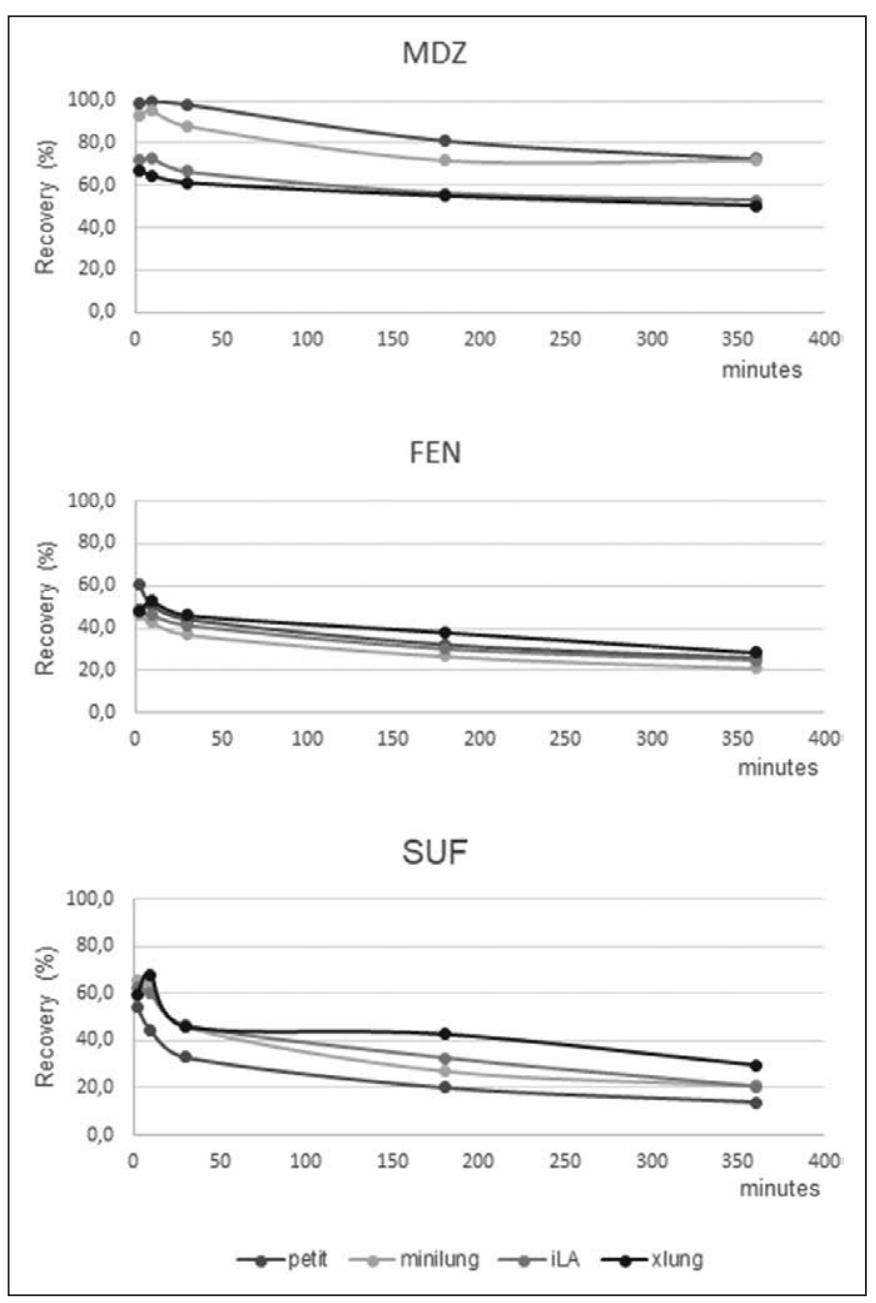

Figure 2. Mean drug recovery (\%) plotted versus time ( $\mathrm{min}$ ) in the first 6 hours for midazolam (MDZ), fentanyl (FEN), sufentanil (SUF) with respect to the four different categories of circuit tested: Minilung petite (dark gray), MiniLung (light gray), iLa Activve (gray), XLung (black).

with Quadrox oxygenators (Maquet Cardiopulmonary AG, Hirrlingen, Germany) showing a similar drug loss of lipophilic drugs. Furthermore, they showed that, besides lipophilicity, PB predicts the amount of drug loss.
Table 2 summarizes the results of the different studies. As shown in Table 2, there are some differences in drug recovery rates between newer ECMO circuits with higher recovery rates of midazolam and fentanyl at 24 hours in our study compared with data published by Shekar et al (34). Both size and circuit components might influence absolute drug adsorption. Overall loss over time seems to be comparable between the studies. This might suggest that the found differences at 24 hours might also be due to study design, priming solutions and albumin concentrations, drug dose, and variability in estimated $t=0$ concentrations and plasma-level variability. It is questionable if the found differences after 24 hours have any clinical consequences.

Fentanyl, sufentanil, and midazolam are all highly lipophilic drugs with $\log P$ values of $3.89,3.4$, and 3.89 , respectively (Table 1, see also Fig. 2 from our previous in vitro article [22]). All have a high PB between $80 \%$ and $97 \%$. Fentanyl has the lowest recovery rate of all the lipophilic drugs. Fentanyl has high levels of both lipophilicity and PB (Table 1), implicating high sequestration rates into the ECMO system and consequently a steep reduction in its plasma concentration $(19,22)$. Its adsorption in the circuit has been previously observed in $\mathrm{CPB}$ (35) and later confirmed also in "in vitro" ECMO setting $(22,34)$. Type of both the oxygenator and the pump seems to influence the degree of sequestration, being the hollow-fiber-based system less prone to adsorb the drug than silicone-based membrane $(21,22)$. As expected, our tests show a dramatic loss of fentanyl, which appears to be uniform across all sizes of circuits tested.

Sufentanil shows a similar pattern as fentanyl with low recovery rates in all circuits. To our knowledge, this is the first study to focus on ECMO-related PK variability of sufentanil. The low recovery rates in the circuit are consistent with drug properties such as the high lipophilicity and high level of $\mathrm{PB}$ (30). Interestingly and in contrast to fentanyl, there are differences in drug recovery between the tested circuits: highest recovery in the biggest circuits compared with the smallest systems. Increase in priming volume is largely affected by increased oxygenator size, which is the primary site of drug adsorption for most drugs (33). Although absolute recovery is

\section{TABLE 2. Comparison of Drug Recovery (\%) Between Study Circuits Versus Different Commercially Available Circuit Designs}

\begin{tabular}{|c|c|c|c|c|c|c|c|c|}
\hline Drugs & $\begin{array}{c}\text { MiniLung } \\
\text { Petite }\end{array}$ & MiniLung & iLA Activve & Xlung & Roller n (16) & Roller ${ }^{a} p$ (16) & Medos $n$ (16) & Quadrox a (34) \\
\hline Midazolam & 58 & 65 & 55.97 & 62 & 0.6 & 0.7 & 63 & 13 \\
\hline Fentanyl & 16 & 10 & 19.57 & 16 & 0.4 & 0.3 & 34 & 3 \\
\hline Morphine & 57 & 45 & 51.29 & 39 & 24 & 30 & 32 & 97 \\
\hline Paracetamol & 47 & 57 & 56.72 & 41 & 34 & 45 & 44 & NA \\
\hline Sufentanil & 18 & 20 & 28.35 & 50 & NA & NA & NA & NA \\
\hline Volume $(\mathrm{mL})$ & 225 & 280 & 360 & 400 & 350 & 900 & 200 & 668 \\
\hline
\end{tabular}

$\mathrm{NA}=$ not applicable.

${ }^{\text {aAt }} 180$ minutes.

Mean drug recovery (in \%) at 24 hours after drug injection, in the current study testing circuits compared with previous in vitro studies. Previous data are related to neonatal roller pump (16), pediatric roller pump (16), neonatal centrifugal pump (16), and adult centrifugal pump (28). 


\section{TABLE 3. Sedoanalgesia During Extracorporeal Membrane Oxygenation: Summary of Physicochemical Properties, In Vitro/Clinical Pharmacologic Data and Related Dose Adjustments of the Main Sedatives and Analgesics}

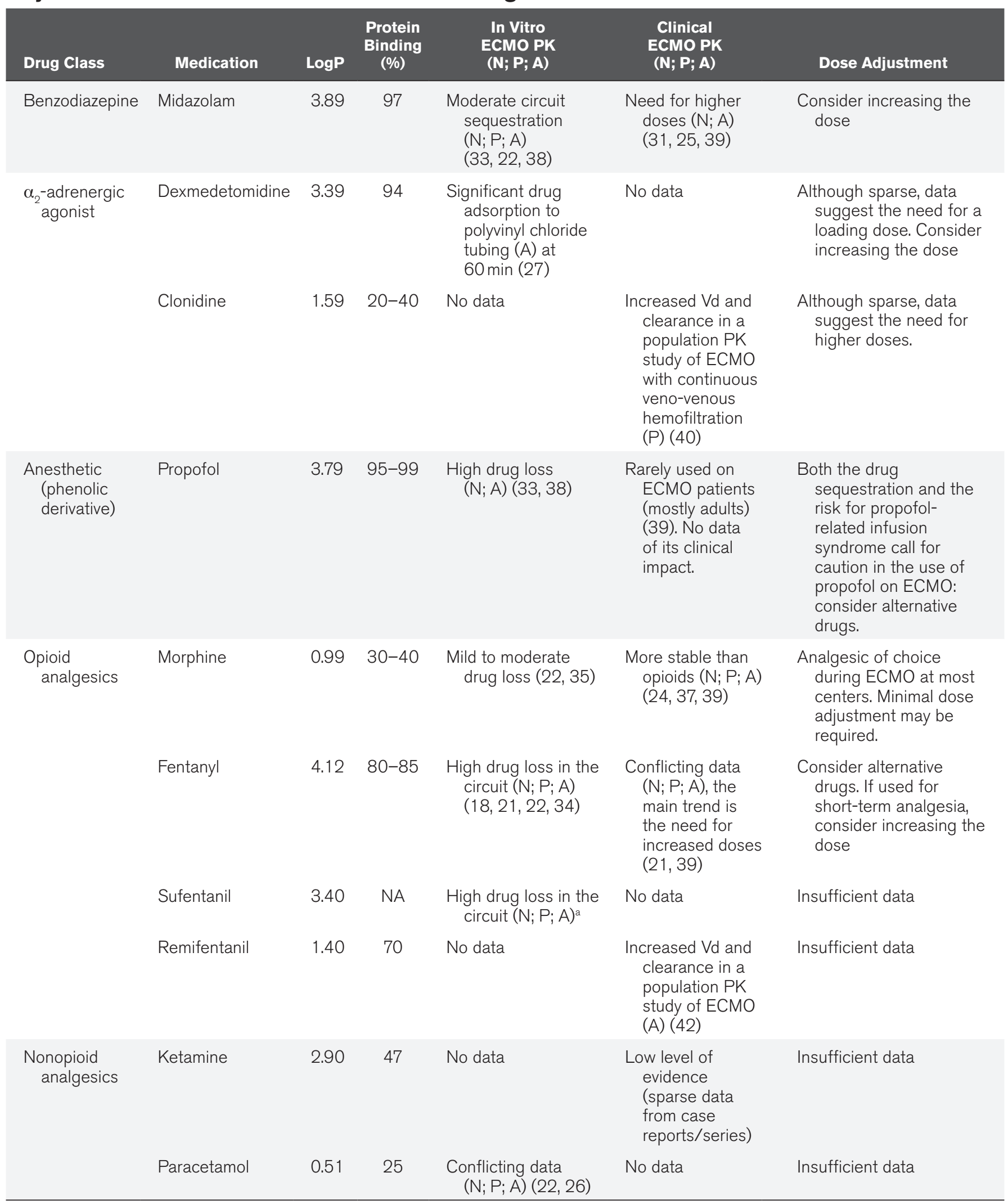

$\mathrm{A}=$ adult, $\mathrm{ECMO}=$ extracorporeal membrane oxygenation, $\mathrm{N}=$ neonatal, $\mathrm{P}=$ pediatric, $\mathrm{PK}=$ pharmacokinetics, $\mathrm{Vd}=$ volume of distribution. a Data derived from current study. 
higher in the largest system, drug loss over time seems to be more or less constant within all systems.

In contrast to sufentanil, midazolam show different recovery rates with the highest adsorption in the largest systems. Although at 24 hours differences are small, initial recovery is highest in the smallest systems. No differences between the tested systems were found for morphine and paracetamol.

Overall circuit size might influence drug adsorption to some extent, but we could not find a clear pattern. Decline over time seems to be comparable between systems with the largest differences in the first samples. Those variations might in part be explained by potential confounding factors such as estimated maximum concentrations as well as potential small sample and dosing errors within the study.

Midazolam shows the highest recovery rate of the lipophilic drugs. Clinical studies show that the Vd of midazolam in ECMOtreated neonates was three- to four-fold greater than in critically ill neonates not on ECMO, resulting in higher daily regimen of sedation (36). The same group documented a decrease in plasma levels in the first 24 hours after cannulation (31). More recently, based on a population PK model of neonates in veno-arterial ECMO, Ahsman et al (25) suggested a potential dosing regimen to provide adequate plasma concentration of midazolam. In the present study, average midazolam drug recovery was $56-65 \%$ in all four systems after 24 hours, which is comparable with our earlier results (22), although higher than reported recovery rates by Shekar et al (35). They also found midazolam recovery to be higher than fentanyl despite similar $\mathrm{PB}$ and $\log \mathrm{P}$ values (35). A clear explanation is currently lacking. Potentially, there are other drug characteristics that influence drug adsorption to some extent. All medication were injected in sequence as it is done in clinical practice. Fentanyl and sufentanil might have a higher affinity to the binding sites than midazolam explaining the differences. Morphine and paracetamol show around 50\% recovery rates in all systems. Concentrations seem to be stable over the 24-hour period and show little variation between systems. These results are in line with our earlier publication (22). There seems to be an initial loss of drugs after injection with no further adsorption over time. Shekar et al (35) found almost no drug loss over time reporting recovery rates close to $100 \%$ in their circuits. This difference is intriguing. In contrast to the lipophilic drugs, a steady state is reached suggesting minimal drug adsorption over time. It is unclear if the initial drug loss is adsorption or an error in estimated maximum concentration. $\mathrm{T}=0$ concentrations are estimated using estimated circulating volume and plasma/whole blood ratio's. Overestimation of the circulating volume might partially explain lower than expected plasma levels found.

Morphine PKs seem to be altered in neonates on ECMO $(24,37)$. Despite this unexplained early drop in plasma levels, their stability over time suggests that morphine and paracetamol are good options for analgesia during ECMO with no or moderate drug loss within the systems.

\section{LIMITATIONS}

Our study has several limitations. In an attempt to simulate the clinical scenario as closely as possible, we tested several drugs at the same time, aware of their possible reciprocal interactions. As it is a single-dose analysis, we were not able to assess if there is a saturation point for drugs' sequestration. Future investigations based on multiple doses/continuous infusion models will help to characterize this aspect. As expected from an ex vivo study design, the analysis is limited to drugs and circuit-related factors, which account only partly for PK variability during ECMO. Patient and diseaserelated factors are not taken into account and were voluntarily excluded, in order to evaluate separately the categories influencing PK on ECMO. However, Table 3 provides a summary of current in vitro $(22,33,35,38)$ and clinical evidence on PK features of the main sedatives and analgesics during ECMO (39-41). Based on drug's physicochemical characteristics, despite the limited level of evidence of the available clinical PK studies, a guidance for pharmacotherapy is suggested. Due to the heterogeneity of the current literature, caution should be paid when interpreting these data: in vitro and in vivo PK knowledge need to be always combined with a strict clinical patient assessment.

Another limit of this study is the significant variation both between identical systems as well as in the control samples. As far as sufentanil PK in the Xlung, T10 sample resulted higher than the T2 sample. The reason remains unclear as the subsequent plasma levels show a similar decreasing pattern. A possible explanation might be a sample error or potential inadequate dispersion at T2 with subsequent lower T10 values, although we are unable to fully explain this "delayed" peak observation.

Due to the closed circuit design, total injected drug volume was kept low, which might introduce a dosing error. Furthermore, total circulating volume was measured after each 24-hour run but could be underestimated due to residual volume within the circuit. Finally, $\mathrm{T}=2-24$ control samples were temperature controlled, but $\mathrm{pH}$ and light exposure were not, which might have increased variability in the control samples.

\section{CONCLUSIONS}

This in vitro study provides a comprehensive body of knowledge into prediction of analgosedative agents PKs during ECMO support with iLA activve circuitry, across all age groups. It highlights the importance of drug factors evaluation (stability, lipophilicity, PB) in relation to circuit design, to adjust therapy while on ECMO. Loss of highly lipophilic drugs in the ECMO circuits at 24 hours was remarkable. There are differences in adsorption of midazolam and morphine between the different commercially available circuits. As optimal dose remains a real challenge in clinical practice, further in vitro laboratory-based research combined to in vivo animal-based PK studies may help to clarify clinical PK variability in order to develop drug dosing guidelines.

\section{ACKNOWLEDGMENTS}

We thank Xenios Germany and Griffini Miglierina Foundation for supporting this study. 


\section{REFERENCES}

1. Gattinoni L, Carlesso E, Langer T: Clinical review: Extracorporeal membrane oxygenation. Crit Care 2011; 15:243

2. DeBerry BB, Lynch JE, Chernin JM, et al: A survey for pain and sedation medications in pediatric patients during extracorporeal membrane oxygenation. Perfusion 2005; 20:139-143

3. Lindén V, Palmér K, Reinhard J, et al: High survival in adult patients with acute respiratory distress syndrome treated by extracorporeal membrane oxygenation, minimal sedation, and pressure supported ventilation. Intensive Care Med 2000; 26:1630-1637

4. Schmidt F, Sasse M, Boehne M, et al: Concept of "awake venovenous extracorporeal membrane oxygenation" in pediatric patients awaiting lung transplantation. Pediatr Transplant 2013; 17:224-230

5. Kress JP, Pohlman AS, O'Connor MF, et al: Daily interruption of sedative infusions in critically ill patients undergoing mechanical ventilation. N Engl J Med 2000; 342:1471-1477

6. Burry L, Rose L, McCullagh IJ, et al: Daily sedation interruption versus no daily sedation interruption for critically ill adult patients requiring invasive mechanical ventilation. Cochrane Database Syst Rev 2014; (7):CD009176

7. Wildschut ED, Hanekamp MN, Vet NJ, et al: Feasibility of sedation and analgesia interruption following cannulation in neonates on extracorporeal membrane oxygenation. Intensive Care Med 2010; 36:1587-1591

8. Ista $\mathrm{E}$, van Dijk M, Gamel C, et al: Withdrawal symptoms in children after long-term administration of sedatives and/or analgesics: A literature review. "Assessment remains troublesome". Intensive Care Med 2007; 33:1396-1406

9. Tobias JD: Tolerance, withdrawal, and physical dependency after long-term sedation and analgesia of children in the pediatric intensive care unit. Crit Care Med 2000; 28:2122-2132

10. Harris J, Ramelet AS, van Dijk M, et al: Clinical recommendations for pain, sedation, withdrawal and delirium assessment in critically ill infants and children: An ESPNIC position statement for healthcare professionals. Intensive Care Med 2016; 42:972-986

11. Wang PP, Huang E, Feng $X$, et al: Opioid-associated iatrogenic withdrawal in critically ill adult patients: A multicenter prospective observational study. Ann Intensive Care 2017; 7:88

12. Kearns GL, Abdel-Rahman SM, Alander SW, et al: Developmental pharmacology-drug disposition, action, and therapy in infants and children. N Engl J Med 2003; 349:1157-1167

13. Allegaert K, Simons SHP, Tibboel D, et al: Non-maturational covariates for dynamic systems pharmacology models in neonates, infants, and children: Filling the gaps beyond developmental pharmacology. Eur J Pharm Sci 2017; 109S:S27-S31

14. Shekar K, Fraser JF, Smith MT, et al: Pharmacokinetic changes in patients receiving extracorporeal membrane oxygenation. J Crit Care 2012; 27:741.e9-741.18

15. Buck ML: Pharmacokinetic changes during extracorporeal membrane oxygenation: Implications for drug therapy of neonates. Clin Pharmacokinet 2003; 42:403-417

16. Wildschut ED, Ahsman MJ, Houmes RJ, et al: Pharmacotherapy in neonatal and pediatric extracorporeal membrane oxygenation (ECMO). Curr Drug Metab 2012; 13:767-777

17. Mulla $\mathrm{H}$, Lawson $\mathrm{G}$, Firmin $\mathrm{R}$, et al: Drug disposition during extracorporeal membrane oxygenation (ECMO). Paediatr Perinat Drug Ther 2001; 4:109-120

18. Mehta NM, Halwick DR, Dodson BL, et al: Potential drug sequestration during extracorporeal membrane oxygenation: Results from an ex vivo experiment. Intensive Care Med 2007; 33:1018-1024

19. Shekar K, Roberts J, McDonald C, et al: Protein bound drugs are prone to sequestration in ECMO circuits: Results from an ex vivo experiment. Crit Care 2015; 19:164

20. Preston TJ, Ratliff TM, Gomez D, et al: Modified surface coatings and their effect on drug adsorption within the extracorporeal life support circuit. J Extra Corpor Technol 2010; 42:199-202

21. Rosen DA, Rosen KR, Silvasi DL: In vitro variability in fentanyl absorption by different membrane oxygenators. J Cardiothorac Anesth 1990; 4:332-335
22. Wildschut ED, Ahsman MJ, Allegaert K, et al: Determinants of drug absorption in different ECMO circuits. Intensive Care Med 2010; 36:2109-2116

23. Leuschen MP, Willett LD, Hoie EB, et al: Plasma fentanyl levels in infants undergoing extracorporeal membrane oxygenation. $J$ Thorac Cardiovasc Surg 1993; 105:885-891

24. Dagan O, Klein J, Bohn D, et al: Effects of extracorporeal membrane oxygenation on morphine pharmacokinetics in infants. Crit Care Med 1994; 22:1099-1101

25. Ahsman MJ, Hanekamp M, Wildschut ED, et al: Population pharmacokinetics of midazolam and its metabolites during venoarterial extracorporeal membrane oxygenation in neonates. Clinic Pharmacokinet 2010; 49:407-419

26. Gillogly A, Kilbourn C, Waldvogel J, et al: In vitro clearance of intravenous acetaminophen in extracorporeal membrane oxygenation. Perfusion 2013; 28:141-145

27. Wagner D, Pasko D, Phillips K, et al: In vitro clearance of dexmedetomidine in extracorporeal membrane oxygenation. Perfusion 2013; 28:40-46

28. Hopfgartner G: Mass spectrometry in bioanalysis-methods, principles and instrumentation. Methods and Principles in Medicinal Chemistry. Weinheim, Germany: Wiley-VCH, 2007; 36, pp 3-62

29. U.S. Department of Health and Human Services Food and Drug Administration: Center for Drug Evaluation and Research (CDER) Center for Veterinary Medicine (CVM). Guidance for industry bioanalytical method validation, 2001. Available at: https://www.fda.gov/ downloads/Drugs/Guidance/ucm070107.pdf. Accessed October 15, 2017

30. Wishart DS, Knox C, Guo AC, et al: DrugBank: A knowledgebase for drugs, drug actions and drug targets. Nucleic Acids Res 2008; 36:D901-D906

31. Mulla H, Lawson G, Peek GJ, et al: Plasma concentrations of midazolam in neonates receiving extracorporeal membrane oxygenation. ASA/O J 2003; 49:41-47

32. Duffett M, Koop A, Menon K, et al: Clonidine for the sedation of critically ill children: A systematic review. J Pediatr Intensive Care 2012; 1:5-15

33. Mulla $\mathrm{H}$, Lawson $\mathrm{G}$, von Anrep $\mathrm{C}$, et al: In vitro evaluation of sedative drug losses during extracorporeal membrane oxygenation. Perfusion 2000; 15:21-26

34. Shekar K, Roberts JA, Mcdonald Cl, et al: Sequestration of drugs in the circuit may lead to therapeutic failure during extracorporeal membrane oxygenation. Crit Care 2012; 16:R194

35. Koren G, Crean P, Klein J, et al: Sequestration of fentanyl by the cardiopulmonary bypass (CPBP). Eur J Clin Pharmacol 1984; 27:51-56

36. Mulla $\mathrm{H}$, McCormack P, Lawson G, et al: Pharmacokinetics of midazolam in neonates undergoing extracorporeal membrane oxygenation. Anesthesiology 2003; 99:275-282

37. Peters JW, Anderson BJ, Simons SH, et al: Morphine metabolite pharmacokinetics during venoarterial extra corporeal membrane oxygenation in neonates. Clin Pharmacokinet 2006; 45:705-714

38. Lemaitre F, Hasni N, Leprince P, et al: Propofol, midazolam, vancomycin and cyclosporine therapeutic drug monitoring in extracorporeal membrane oxygenation circuits primed with whole human blood. Crit Care 2015; 19:40

39. Shekar K, Roberts JA, Mullany DV, et al: Increased sedation requirements in patients receiving extracorporeal membrane oxygenation for respiratory and cardiorespiratory failure. Anaesth Intensive Care 2012; 40:648-655

40. Kleiber N, Mathôt RA, Ahsman MJ, et al: Population pharmacokinetics of intravenous clonidine for sedation during paediatric extracorporeal membrane oxygenation and continuous venovenous hemofiltration. Brit J Clin Pharmacol. 2017; 83:1227-1239

41. Franck LS, Vilardi J, Durand D, et al: Opioid withdrawal in neonates after continuous infusions of morphine or fentanyl during extracorporeal membrane oxygenation. Am J Crit Care 1998; 7:364-369

42. Yang S, Noh H, Hahn J, et al: Population pharmacokinetics of remifentanil in critically ill patients receiving extracorporeal membrane oxygenation. Sci Rep 2017; 7:16276 